\title{
Review of: "Identification of an isoflavonoid transporter required for the nodule establishment of the Rhizobium-Fabaceae symbiotic interaction"
}

\author{
ANDREA SQUARTINI ${ }^{1}$ \\ 1 University of Padua \\ Potential competing interests: The author(s) declared that no potential competing interests exist.
}

This is a well-conceived and clearly written report that can cast light on important aspects of the molecular plant-microbe interactions in nitrogen-fixing symbioses. I have some points that require the authors' attention, which can be outlined as follows.

LaMATE2 silencing basically reduces to half the genistein release (Fig.5b) This appears a somewhat partial effect of the silencing considering that it was reported to be as high as $80 \%$ under $\mathrm{N}$-deficient conditions. Does this mean that the flavonoid could also permeate across the membrane without the need of its specific transporter with a kinetics that appears nevertheless $50 \%$ as efficient ? Is the observed export consistent with LAMATE2 being the only mechanism for genistein efflux? Or, could there possibly be other transporters (unknown and intact) that would be responsible for the residual excretion of this flavonoid? As flavonoids are overall hydrophobic compounds, if a specific transporter would be a necessary component of their export, how would it compete with the plant membrane itself and with the fact that flavonoids would have the tendency to just partition into membranes rather than being attracted/conveyed to a given transporter?

To frame this aspect and better support the concept that there would be a strict need for a flavonoid transporter, the authors could discuss also what is known for the Bradyrhizobium side of the transport. The exported genistein needs to enter the rhizobial cells as well by passing their membranes and reaching a target (supposedly a NodD protein regulator?). Is there any evidence of a corresponding receptor/transporter in the membrane(s) (outer and inner) of the gram-negative Bradyrhizobium cell ? Or is the bacterial trafficking known to rely on a mere partitioning into their cells' membranes or binding the nod genes regulator there? However that bacterial regulator protein would then need to reach the nod box operons to activate the nod gene expression. The challenging underlying question here being, are transporters really needed for flavonoids to cross plant membranes (outbound) and then bacterial membranes (inbound)?

How is the rather different level of expression silencing under $\mathrm{N}$ - or P-deficiency explained considering that the silencing technique is the same?

Why is an $80 \%$ silencing (in $\mathrm{N}$-deficiency) leading to much less effective reduction of genistein release than a $60 \%$ silencing (P-deficiency) ? 
Why is the exogenous genistein complementation of the silenced gene (Fig. 6b), with a supposedly nonlimiting concentration, not fully restoring the nodule number? In the yeast vescicles experiment the specificity of transport for genistein over other flavonoids and compounds appears clear, but could this be due to the fact that genistein would be particularly prone to be docked and transported by proteins in general ? Besides the empty vector, would a different cloned protein (of similar hydrophobicity, membrane topology but different sequence) have no effect ? Minor points:

Line 78. Phosphorous is the adjective, phosphorus the noun, which is meant here Line 96: . Around 100 Mio years ago. (Mio ?)

Line 148 "particularly root structures, called cluster roots..." particular root structures 589 "During the last years, we learned a lot about the signaling pathways..." language is too colloquial 